ANNALES

POLONICI MATHEMATICI

$87(2005)$

\title{
Reduction of semialgebraic constructible functions
}

\author{
by LUDWIG BRÖCKER (Münster)
}

In memory of Stanistaw Lojasiewicz (1926-2002)

\begin{abstract}
Let $R$ be a real closed field with a real valuation $v$. A $\mathbb{Z}$-valued semialgebraic function on $R^{n}$ is called algebraic if it can be written as the sign of a symmetric bilinear form over $R\left[X_{1}, \ldots, X_{n}\right]$. We show that the reduction of such a function with respect to $v$ is again algebraic on the residue field. This implies a corresponding result for limits of algebraic functions in definable families.
\end{abstract}

Introduction. One of the most fundamental results in real algebraic geometry is the theorem of Tarski-Seidenberg, stating that if $S \subset \mathbb{R}^{n}$ is semialgebraic and $f: \mathbb{R}^{n} \rightarrow \mathbb{R}^{k}$ is a polynomial map, then $f(S)$ is also semialgebraic.

However, if $S$ is algebraic, then in general $f(S)$ is no longer so. The simplest example is where $S=\left\{x \in \mathbb{R}^{2} \mid x_{1}^{2}+x_{2}^{2}=1\right\}$, the unit circle in $\mathbb{R}^{2}$, and $f$ is the projection onto the first coordinate in $\mathbb{R}^{2}$. Then $f(S)$ is the unit ball $\left\{x \in \mathbb{R} \mid x^{2} \leq 1\right\}$ in $\mathbb{R}$ which is obviously not algebraic. So the consideration of images is too rough in order to save information on the algebraicity of $S$.

Also, let $\varphi: \mathbb{R}^{n} \rightarrow \mathbb{Z}$ be a constructible function, that is, $\varphi\left(\mathbb{R}^{n}\right)$ is finite and $\varphi^{-1}(z)$ is semialgebraic for all $z \in \mathbb{Z}$. Let again $f: \mathbb{R}^{n} \rightarrow \mathbb{R}^{k}$ be a polynomial map. It is not a priori clear how to define the image function $f_{*} \varphi: \mathbb{R}^{k} \rightarrow \mathbb{Z}$, but it turns out that a well behaved definition is

$$
f_{*} \varphi(x)=\int_{f^{-1}(x)} \varphi d \chi,
$$

where the right hand side is the so-called Euler integral, to be explained in Section 1. This extension of the classical Euler characteristic to constructible functions appeared in many contexts.

2000 Mathematics Subject Classification: 14P10, 12J25, 28A25.

Key words and phrases: limits of semialgebraic functions, reduction with respect to a valuation, Euler integral. 
Now let $\varphi=1_{S}$ where $S$ is algebraic. Then $f_{*} \varphi$ is algebraic in the following sense. There exist finitely many polynomials $f_{1}, \ldots, f_{m} \in \mathbb{R}\left[X_{1}, \ldots X_{k}\right]$ such that $f_{*} \varphi(x)=\operatorname{sign} f_{1}(x)+\cdots+\operatorname{sign} f_{m}(x)$ for all $x \in \mathbb{R}^{k}$. In the example where $S$ is the unit circle in $\mathbb{R}^{2}$, we have $f_{*} \varphi(x)=2$ for $x^{2}<1, f_{*} \varphi(x)=1$ for $x^{2}=1$ and $f_{*} \varphi(x)=0$ for $x^{2}>1$. Thus $f_{*} \varphi=\operatorname{sign} f_{1}+\operatorname{sign} f_{2}$ for $f_{1}=1-X^{2}$ and $f_{2} \equiv 1$.

More generally, a beautiful theorem of Parusiński-Szafraniec [P-S] states that if $\varphi: \mathbb{R}^{n} \rightarrow \mathbb{Z}$ is algebraic, then so is $f_{*} \varphi$.

We will show a corresponding result for limits in semialgebraic families, where one has a similar situation:

Let $S \subset \mathbb{R}^{n} \times \mathbb{R}^{k}$ be semialgebraic. For $t \in \mathbb{R}^{k}$ let $S_{t}:=S \cap \mathbb{R}^{n} \times\{t\}$. Let $\left(t_{m}\right) \in \mathbb{R}^{k}$ be a sequence such that $\left(S_{t_{n}}\right)$ tends to $T$ in the Hausdorff sense. It is known that $T$ is again semialgebraic [Br1], [Br2], which holds correspondingly in the o-minimal context [L-S], [vdD1], [vdD2]. Again, if $S$ is algebraic, this need not be the case for $T$.

For instance, consider the family of ellipses $E_{t} \subset \mathbb{R}^{2}$ where

$$
E_{t}=\left\{x \in \mathbb{R}^{2} \mid x_{1}^{2}+t^{2} x_{2}^{2}=1\right\},
$$

and let $\left(t_{n}\right) \rightarrow \infty$. Then $\left(E_{t_{n}}\right) \rightarrow\left\{x \in \mathbb{R}^{2} \mid x_{1}^{2} \leq 1, x_{2}=0\right\}$, which is not algebraic.

So again, Hausdorff limits are not appropriate. In Section 6 we will introduce a different kind of convergence using local Euler integrals, which extends to constructible functions. Then we will show that the limit of a sequence of constructible functions in an algebraic family is again algebraic (Theorem 6.4). The main ingredient is our Theorem 5.1 which expresses the situation in terms of valuations and reduction maps.

1. Euler integral. Let $R$ be a real closed field and let $\omega$ be the class of all semialgebraic sets $S \subset R^{n}$ for some $n$. A function $\varphi: R^{n} \rightarrow \mathbb{Z}$ is called constructible if the range of $\varphi$ is finite and $\varphi^{-1}(z)$ is semialgebraic for all $z \in \mathbb{Z}$.

For any semialgebraic set $S \subset R^{n}$ the Euler characteristic (with compact supports) $\chi(S)$ is defined. If $S$ is semialgebraically isomorphic to an open $d$-cell, then $\chi(S)=(-1)^{d}$. For arbitrary $S$, this allows us to compute $\chi(S)$ from a cell decomposition, which always exists, and it turns out that the computation of $\chi(S)$ does not depend on the decomposition. One can extend the Euler characteristic to constructible functions as follows (compare [V]). Let $\varphi=\sum a_{i} 1_{S_{i}}$ where the sum is finite, $a_{i} \in \mathbb{Z}$ and $R^{n} \supset S_{i}$ is semialgebraic. Then $\chi(\varphi):=\sum a_{i} \chi\left(S_{i}\right)$. Again, this is independent of the representation of $\varphi$. 
One also writes

$$
\chi(\varphi)=\int \varphi d \chi=\int_{R^{n}} \varphi(x) d \chi(x),
$$

and if $T \subset R^{n}$ is semialgebraic, then

$$
\int_{T} \varphi d \chi:=\int_{R^{n}} 1_{T} \cdot \varphi d \chi
$$

this is called Euler integration. This name is justified by the following rules:

(i) If $\varphi, \psi: R^{n} \rightarrow \mathbb{Z}$ are constructible, then $\int(\varphi+\psi) d \chi=\int \varphi d \chi+\int \psi d \chi$.

(ii) If, moreover, $f: R^{n} \rightarrow R^{m}$ is a continuous semialgebraic map, then

$$
\int_{R^{n}} \varphi(x) d \chi(x)=\int_{R^{m}}\left(\int_{f^{-1}(y)} \varphi(x) d \chi(x)\right) d \chi(y)
$$

(Fubini-Cavalieri).

In the situation of (ii) we define $f_{*} \varphi: R^{m} \rightarrow \mathbb{Z}, y \mapsto \int_{f^{-1}(y)} \varphi(x) d \chi(x)$. Thus

$$
\int_{R^{n}} \varphi(x) d \chi(x)=\int_{R^{m}} f_{*} \varphi(y) d \chi(y)
$$

2. Algebraic functions. Again, let $R$ be a real closed field.

Definition 2.1. A constructible function $\varphi: R^{n} \rightarrow \mathbb{Z}$ is called algebraic if there are finitely many polynomials $p_{1}, \ldots, p_{k} \in R[X], X=\left(X_{1}, \ldots, X_{n}\right)$, such that $\varphi(x)=\operatorname{sign}\left(p_{1}(x)\right)+\cdots+\operatorname{sign}\left(p_{n}(x)\right)$ for all $x \in R^{n}$.

EXAMPLE 2.2. Let $V \subset R^{n}$ be a real algebraic set. Then $1_{V}$ is algebraic. In fact, there is a positive polynomial $p$ for $V$, that is, $p(x)>0$ for $x \notin V$ and $p(x)=0$ for $x \in V$. Now $1_{V}=\operatorname{sign}(1)+\operatorname{sign}(-p)$.

REMARK 2.3. Let $\varphi, \psi: R^{n} \rightarrow \mathbb{Z}$ be algebraic. Then

(a) $\varphi+\psi$ and $\varphi \cdot \psi$ are algebraic.

(b) Let $V \subset R^{n}$ be an algebraic subset. Then $\zeta$ is algebraic, where $\zeta(x)=$ $\varphi(x)$ for $x \notin V$ and $\zeta(x)=\psi(x)$ for $x \in V$.

(c) Let $S$ be a symmetric $n \times n$ matrix with coefficients in $R[X]$. Then $x \mapsto \operatorname{sign}_{x} S$ (signature of $S$ at $x$ ) is algebraic.

Here (a) is obvious, (b) follows easily from (a) and Example 2.2, and (c) follows from (b) by induction on the dimension, since we can diagonalize $S$ over function fields, that is, up to algebraic sets of smaller dimensions.

The fundamental property of algebraic functions is (see $[\mathrm{P}-\mathrm{S}]$ ):

Theorem 2.4 (Parusiński-Szafraniec). Let $f: R^{n} \rightarrow R^{m}$ be a polynomial map. If $\varphi: R^{n} \rightarrow \mathbb{Z}$ is algebraic, then so is $f_{*} \varphi: R^{m} \rightarrow \mathbb{Z}$. 
We are going to show a corresponding result for reduction maps. This requires more preparations (for more details see [B-C-R, Chap. 7], [A-B-R, Chap. 2]):

Let $V=\operatorname{Spec}(A)$ be an algebraic variety over $R$. Then $\operatorname{Hom}(A, R)=$ $V(R)$ is the variety of closed real points in $V$. We extend $V(R)=\operatorname{Hom}(A, R)$ to the space $\operatorname{Spec}_{\mathrm{r}}(A)=\left\{\alpha: A \rightarrow R_{\alpha}\right\} / \sim$ called the real spectrum of $A$, where $R_{\alpha}$ is real closed, $\alpha$ is a homomorphism and $\sim$ is generated by commutative triangles

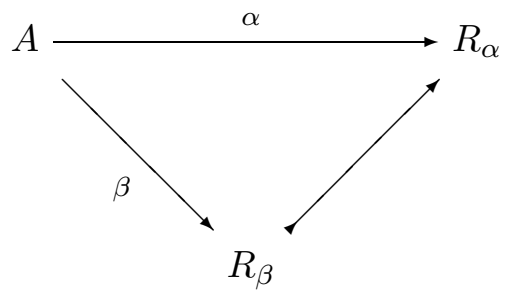

From the model completeness of the theory of real closed fields it is clear that elementary objects and properties in $V(R)$ and $\operatorname{Spec}_{\mathrm{r}}(A)$ correspond to each other. For instance, a constructible (algebraic) function $\varphi$ on $V(R)$ extends uniquely to a constructible (algebraic) function $\widetilde{\varphi}$ on $\operatorname{Spec}_{\mathrm{r}}(A)$.

Let $\alpha \in \operatorname{Spec}_{\mathrm{r}}(A)$. Then $\operatorname{Ker}(\alpha)=: \operatorname{supp}(\alpha)$ (support of $\alpha$ ) is a prime ideal of $A$. We define $\operatorname{dim}(\alpha):=\operatorname{dim}(A / \operatorname{supp}(\alpha))$. One defines $\operatorname{Spec}_{\mathrm{r}}(A)$ correspondingly for any commutative ring with unit, and also constructible and algebraic functions. For instance, if $A=K$ is a field, then $\operatorname{Spec}_{\mathrm{r}}(K)$ is the space of all orderings of $K$. The representation theorem [Be-Br] provides a criterion for the algebraicity of a constructible function $\varphi: \operatorname{Spec}_{\mathrm{r}}(K) \rightarrow \mathbb{Z}$. For general $A$ one has

$$
\operatorname{Spec}_{\mathrm{r}}(A)=\bigcup_{p \in \operatorname{Spec}(A)} \operatorname{Spec}_{\mathrm{r}}(k(p))
$$

where $k(p)$ is the residue field of $p \in \operatorname{Spec}(A)$. This allows us to reduce questions about $V(R)$, via $\operatorname{Spec}_{\mathrm{r}}(A)$, to features and tools of the real algebra of fields. We will follow this path in the next sections.

3. The reduction map. Throughout this section we fix a real closed field $R$ together with a real valuation $v: R \rightarrow \Gamma \cup \infty$, where $\Gamma$ is the value group. We denote by $B \subset R$ the valuation ring, by $m$ the maximal ideal and by $\bar{R}$ the residue field. Note that $v$ is henselian, $\Gamma$ is divisible and $\bar{R}$ is again real closed. The residue map $\varrho: B \rightarrow \bar{R}, b \mapsto \varrho(b)=: \bar{b}$, admits a section $\sigma: \bar{R} \rightarrow B$ which we fix in the following. So we consider $\bar{R}$ as a subfield of $R$. We denote by $\varrho$ also the map $B^{n} \rightarrow \bar{R}^{n}, x_{i} \mapsto \varrho\left(x_{i}\right), i=1, \ldots, n$. One has [Br1, Th. 1.7] 
Proposition 3.1. Let $S \subset B^{n}$ be definable in the language of valued ordered fields. Then $\varrho(S) \subset \bar{R}^{n}$ is semialgebraic.

Again, we want to extend $\varrho$ to constructible functions. For this, let $\varphi$ : $R^{n} \rightarrow \mathbb{Z}$ be constructible, as in Section 1 , and let $a \in B^{n}$. For $\lambda \in R, \lambda>0$ let

$$
D(a, \lambda):=\left\{x \in R^{n} \mid(a-x)_{i}<\lambda \text { for } i=1, \ldots, n\right\}
$$

and

$$
\chi(a, \lambda)(\varphi):=\int_{D(a, \lambda)} \varphi d \chi .
$$

The map $] 0, \infty[\rightarrow \mathbb{Z}, \lambda \mapsto \chi(a, \lambda)(\varphi)$, is constructible. Hence there exist $0<\lambda_{1}<\lambda_{2}, \lambda_{1} \in m, \lambda_{2} \in B \backslash m$, such that $\chi(a, \lambda)(\varphi)$ is constant on ]$\lambda_{1}, \lambda_{2}[$, so we define

$$
\chi(a, m)(\varphi):=\int_{\varrho^{-1}(\bar{a})} \varphi d \chi:=\int_{D(a, \lambda)} \varphi d \chi
$$

for $\lambda \in] \lambda_{1}, \lambda_{2}[$ and $\bar{a}=\varrho(a)$. Then we get

Proposition 3.2. Let $\varphi: R^{n} \rightarrow \mathbb{Z}$ be constructible. Then so is $\varrho_{*} \varphi:$ $\bar{R}^{n} \rightarrow \mathbb{Z}$, where for $\bar{a} \in \bar{R}^{n}$,

$$
\varrho_{*} \varphi(\bar{a}):=\int_{\varrho^{-1}(\bar{a})} \varphi d \chi
$$

Proof. For all $z \in \mathbb{Z}$ the set $\left\{a \in B^{n} \mid \chi(a, m)(\varphi)=z\right\}=: e(z, \varphi)$ is definable in the language of valued fields. By Hardt's theorem [Ha] it is also clear that $\chi(a, m)(\varphi)$ is bounded for fixed $\varphi$ and $a \in B^{n}$. So we can write

$$
\chi(a, m)(\varphi)=\sum z_{i} 1_{e\left(z_{i}, \varphi\right)}
$$

where the sum is finite. Clearly every function $1_{e\left(z_{i}, \varphi\right)}$ is constant on the fibers of $\varrho$. Hence

$$
\varrho_{*} \varphi=\sum z_{i} 1_{\varrho\left(e\left(z_{i}, \varphi\right)\right)},
$$

which by Proposition 3.1 is constructible.

4. The reduction map on the real spectrum. In Section 2 we saw that to every constructible function $\varphi: R^{n} \rightarrow \mathbb{Z}$ there corresponds a constructible function $\widetilde{\varphi}: \operatorname{Spec}_{\mathrm{r}}(A) \rightarrow \mathbb{Z}$, where $A=R\left[X_{1}, \ldots, X_{n}\right]$. If, in particular, $S$ is semialgebraic and $\varphi=1_{S}$ then $\widetilde{\varphi}=1_{\widetilde{S}}$, the constructible set $\widetilde{S} \subset \operatorname{Spec}_{\mathrm{r}}(A)$ being defined by the same equations as $S$. The set $B^{n} \subset R^{n}$ is not semialgebraic. Nevertheless, we define

$$
\widetilde{B}^{n}=\left\{\alpha \in \operatorname{Spec}_{\mathrm{r}}(A) \mid \forall f \in A \text { with } \alpha(f)>0 \exists x \in B^{n} \text { with } f(x)>0\right\} .
$$


We are going to study a reduction

$$
\widetilde{\varrho}: \widetilde{B}^{n} \rightarrow \operatorname{Spec}_{\mathrm{r}}(\bar{A}),
$$

where $\bar{A}=\bar{R}\left[X_{1}, \ldots, X_{n}\right]$. The constructions and results below are taken from [Br1, §3]. Let $\alpha \in \widetilde{B}^{n}$. Recall that $\alpha$ corresponds to an ultrafilter, say $\varphi(\alpha)$, of semialgebraic sets in $R^{n}$. Then the sets $\varrho\left(S \cap B^{n} \mid S \in \varphi(\alpha)\right)$ generate a unique ultrafilter $\varphi^{\prime}$ of semialgebraic sets in $\bar{R}^{n}$. So we define $\widetilde{\varrho}(\alpha)$ to be the corresponding element in $\operatorname{Spec}_{\mathrm{r}}(\bar{A})$, that is, $\varphi^{\prime}=\varphi(\widetilde{\varrho}(\alpha))$.

Now let $V \subset R^{n}$ be a closed integral algebraic subvariety with $\operatorname{dim}(V)$ $=k$, and let $\bar{V}_{1}, \ldots, \bar{V}_{r}$ be the irreducible components of $\mathrm{cl}_{\mathrm{z}} \varrho\left(V \cap B^{n}\right.$ ) (where $\mathrm{cl}_{\mathrm{z}}$ denotes the Zariski closure), for which $\operatorname{dim} \bar{V}_{i}=k$. For each $i=1, \ldots, r$ we shall define a set $v_{i j}$ of valuations of $R(V)$ which extend the given valuation $v$ on $R$ in a natural way. For this let

$$
B[V]:=\{f+I(V) \in R[V] \mid f \in B(X)\}
$$

and

$$
p_{i}:=\left\{f+I(V) \in R[V] \mid f \in B[X] \text { and } \bar{f}(x)=0 \forall x \in \bar{V}_{i}\right\} .
$$

Here $X=\left(X_{1}, \ldots, X_{n}\right), x=\left(x_{1}, \ldots, x_{n}\right)$ and $\bar{f}$ is the componentwise reduction of $f$. Then $p_{i}$ is a prime ideal in $B[V]$ and $B[V] / p_{i} \simeq \bar{R}\left[\bar{V}_{i}\right]$. Let $A_{i}=B[V]_{p_{i}}$ and $\widetilde{A}$ the integral closure of $A_{i}$ in $R(V)$. In general, $A_{i} \neq \widetilde{A}_{i}$ and also $\widetilde{A}_{i}$ is possibly not a valuation ring in $R(V)$. However, one has

Proposition 4.1. Under the above notations, $\widetilde{A}_{i}$ is semilocal with maximal ideals $m_{i j}, j=1, \ldots, s(i)$, where $m_{i j} \cap B[V]=p_{i}$. The localization $\left(\widetilde{A}_{i}\right)_{m_{i j}}$ is the valuation ring of a valuation $v_{i j}$ of $R(V)$ extending $v$. The residue field of $v_{i j}$ is a finite extension of $\bar{R}\left(\bar{V}_{i}\right)$ and the value group of $v_{i j}$ is divisible for $j=1, \ldots, s(i)$.

Quite generally, an ordering $\alpha$ of a field $K$ is called compatible with a valuation $v$ of $K$ if the valuation ring of $v$ is convex with respect to $\alpha$. In that case $\alpha$ induces an ordering $v(\alpha)$ on the residue field. With these notations we have moreover:

Proposition 4.2. The ordering $\alpha \in \operatorname{Spec}_{\mathrm{r}}(R(V))$ is compatible with one of the valuations $v_{i j}$ if and only if $\alpha \in \widetilde{B}^{n}$ and $\operatorname{dim}(\widetilde{\varrho}(\alpha))=\operatorname{dim}(\alpha)=$ $\operatorname{dim}(V)$. In that case $\widetilde{\varrho}(\alpha) \in \operatorname{Spec}_{\mathrm{r}}\left(\bar{R}\left(\bar{V}_{i}\right)\right)$ and $v_{i j}(\alpha)$ extends $\widetilde{\varrho}(\alpha)$.

Finally, in the above situation, we would like to have some information on the number of those $\alpha \in \operatorname{Spec}_{\mathrm{r}}(R(V))$ for which $\widetilde{\varrho}(\alpha)=\bar{\alpha} \in \operatorname{Spec}_{\mathrm{r}}\left(\bar{R}\left(\overline{V_{i}}\right)\right)$. Here we have

Proposition 4.3. Under the above notations, for $i=1, \ldots, r$ there exists a nondegenerate quadratic form $\varphi_{i}$ over $\bar{R}\left(\bar{V}_{i}\right)$ such that for all $\bar{\alpha} \in$ 
$\operatorname{Spec}_{\mathrm{r}}\left(\bar{R}\left(\bar{V}_{i}\right)\right)$ one has

$$
\#\left\{\alpha \in \operatorname{Spec}_{\mathrm{r}}(R(V)) \mid \widetilde{\varrho}(\alpha)=\bar{\alpha}\right\}=\operatorname{sign}_{\bar{\alpha}}\left(\varphi_{i}\right) .
$$

In fact, $\varphi_{i}$ is the trace form of $\widetilde{A}_{i} / p_{i} \widetilde{A}_{i}$ over $\bar{R}\left(\bar{V}_{i}\right)$.

More generally, let $g \in B[V]$ and for $x, y \in \widetilde{A}_{i} / p_{i} \widetilde{A}_{i}$ let $\varphi_{i}(g)(x, y):=$ $\operatorname{tr} g x y$, where tr is again the trace of $\widetilde{A}_{i} / p_{i} \widetilde{A}_{i}$ over $\bar{R}\left(\bar{V}_{i}\right)$.

Proposition 4.4. For $\bar{\alpha} \in \operatorname{Spec}_{\mathrm{r}}\left(\bar{R}\left(\bar{V}_{i}\right)\right)$ one has

$$
\begin{aligned}
\operatorname{sign}_{\bar{\alpha}}\left(\varphi_{i}(g)\right)= & \#\left\{\alpha \in \operatorname{Spec}_{\mathrm{r}}(R(V)) \mid \widetilde{\varrho}(\alpha)=\bar{\alpha}, \alpha(g)>0\right\} \\
& -\#\left\{\alpha \in \operatorname{Spec}_{\mathrm{r}}(R(V)) \mid \widetilde{\varrho}(\alpha)=\bar{\alpha}, \alpha(g)<0\right\} .
\end{aligned}
$$

REMARK 4.5. Propositions 4.3 and 4.4 also hold if $V$ is not necessarily integral, but merely reduced. Consider the following situation: $\bar{W} \subset \bar{R}^{n}$ is an irreducible variety. If we fix a section $\bar{R} \hookrightarrow R$, then $\bar{W}$ corresponds to a subvariety $W$, again irreducible, of $R^{n}$. Consider $R \times W \subset R^{n}$ (possibly replace $n$ by $n+1)$, let $f \in R[R \times W]$ and let $V=Z(f)$ be the zero set of $f$. Assume that $\bar{W}$ coincides with one of the $\bar{V}_{i}$, say $\bar{W}=\bar{V}_{1}$. Assume further, for simplicity, that $f$, regarded as a polynomial over $R(W)$, is separable. Now let $\bar{\alpha} \in \operatorname{Spec}_{\mathrm{r}}(\bar{R}(\bar{W}))$. There is a unique $\alpha \in \operatorname{Spec}_{\mathrm{r}}(R(W))$ with $\widetilde{\varrho}(\alpha)=\bar{\alpha}$. Consider the corresponding real closed field $R_{\alpha} \supset R(W)$ and the valuation ring $B_{\alpha} \subset R_{\alpha}$ where

$$
B_{\alpha}=\left\{x \in R_{\alpha} \mid \exists b \in B: x \leq b\right\} .
$$

Also let $m_{\alpha}$ be the corresponding maximal ideal. Then the zeros of $f$ in $B_{\alpha}$ correspond to those $\alpha \in \operatorname{Spec}_{\mathrm{r}}(R(V))$ which are compatible with a valuation $v_{i j}$ and for which $\widetilde{\varrho}(\alpha)=\bar{\alpha}$. Moreover, $\alpha \in m_{\alpha}$ if and only if $\alpha$ is compatible with a valuation $v_{1 j}$.

5. Reduction of algebraic functions. In this section we show our main result:

ThEOREM 5.1. Let $\varphi: R^{n} \rightarrow \mathbb{Z}$ be algebraic. Then so is $\varrho_{*} \varphi: \bar{R}^{n} \rightarrow \mathbb{Z}$.

Here we use the notations of Section 3. For the proof we will need several steps and the lemma below which is essentially [P-S, Lemma 6], but note that unlike that article we always consider the Euler characteristic with compact supports.

LEMMA 5.2. Let $R$ be a real closed field, $a<b \in R$ and $f=a_{d} X^{d}+\cdots$ $+a_{0}, a_{d} \neq 0$, a polynomial such that $f(a), f(b) \neq 0$. Moreover, for $2 \leq k \leq d$ let

$$
V_{k}:=\left\{x \in R \mid f(x)=\cdots=f^{(k-1)}(x)=0\right\}
$$

and

$$
Z_{k, a, b}:=\sum_{x \in V_{k}, a<x<b} \operatorname{sign}\left(f^{(k)}(x)\right) .
$$


Then

$$
\int_{] a, b[} \operatorname{sign}(f) d \chi=-\frac{1}{2}(\operatorname{sign}(f(a))+\operatorname{sign}(f(b)))-\sum_{2 \leq 2 k \leq d} Z_{2 k, a, b} .
$$

Proof of Theorem 5.1. First of all, we may assume that $\varphi(x)=\operatorname{sign}(f(x))$ for all $x \in R^{n}$, where $f$ is a polynomial $\in R\left[X_{1}, \ldots, X_{n}\right]$.

1. Without changing $\varphi=\operatorname{sign}(f)$ we may assume that $f \in B\left[X_{1}, \ldots, X_{n}\right]$, but $f \notin m\left[X_{1}, \ldots, X_{n}\right]$. Let $\bar{f} \in \bar{R}\left[X_{1}, \ldots, X_{n}\right]$ be the componentwise reduction of $f$, so $\bar{f} \neq 0$. Clearly, $\varrho_{*} \varphi(y)=\operatorname{sign} \bar{f}(y)$ for all those $y \in \bar{R}^{n}$ for which $\bar{f}(y) \neq 0$. In other words, $\varrho^{*} \varphi$ is algebraic up to a set of smaller dimension.

2. Now let $W^{\prime} \subset \bar{R}^{n}$ be an algebraic variety such that the following hold:

- There is an algebraic function $\varphi^{\prime}: \bar{R}^{n} \rightarrow \mathbb{Z}$ with $\varrho_{*} \varphi=\varphi^{\prime}$ on $\bar{R}^{n} \backslash W^{\prime}$.

- $W^{\prime}$ is of minimal dimension and has a minimal number of components subject to the above property.

We want to show that $W^{\prime}=\emptyset$. Assume $W^{\prime} \neq \emptyset$. So let $\bar{W}$ be a component of $W^{\prime}$ with $\operatorname{dim} \bar{W}=\operatorname{dim} W^{\prime}$. We will show

3. $\varrho_{*} \varphi$ is generically algebraic on $\bar{W}$ (algebraic up to a set of smaller dimension). By Remark 2.3 this would contradict $W^{\prime} \neq \emptyset$. As before, we fix a section $\bar{R} \hookrightarrow B$. Thus $\bar{W}$ corresponds to a subvariety $W$ of $R^{n}$. Let $N$ be the normal bundle of $W$ in $R^{n}$ :

$$
N=\left\{(x, a) \mid x \in R^{n}, a \in W, x \perp T_{a} W\right\} .
$$

Here $T_{a} W$ is the tangent space of $W$ at $a$. (If $a$ is a singular point, then $T_{a} W=R^{n}$.) Similarly, we have

$$
\bar{N}=\left\{(y, b) \mid y \in \bar{R}^{n}, b \in \bar{W}, y \perp T_{b} \bar{W}\right\} .
$$

Let $\bar{r}: \bar{W} \rightarrow \bar{R}$ be a polynomial map such that $\bar{r}(b)>0$ if $b$ is nonsingular and $\bar{r}(b)=0$ if $b$ is singular. Let

$$
\begin{aligned}
& \bar{U}_{\bar{r}}:=\{(y, b) \in \bar{N} \mid\langle y, y\rangle<\bar{r}(b)\}, \\
& U_{r}:=\{(x, a) \in N \mid\langle x, x\rangle<r(a)\},
\end{aligned}
$$

where $\langle$,$\rangle denotes the usual scalar product and where, by the identification$ of $\bar{R}$ with a subfield of $R$, we may consider $\bar{r}$ as a polynomial map $r$ on $W$. Let

$$
\begin{array}{ll}
\pi: N \rightarrow R^{n}, & (x, a) \mapsto x+a, \\
\bar{\pi}: \bar{N} \rightarrow \bar{R}^{n}, \quad(y, b) \mapsto y+b .
\end{array}
$$

We may choose $\bar{r}$ in such a way that $\pi$ and $\bar{\pi}$ map $U=U_{r}$ (and $\bar{U}=\bar{U}_{r}$ ) isomorphically onto a neighbourhood of $W_{\text {reg }}$ (and $\bar{W}_{\text {reg }}$ respectively). We have

$$
r_{*} \varphi(y)=\int_{r^{-1}(y)} \varphi d \chi=\int_{\pi^{-1} r^{-1}(y) \cap U} \pi^{*} \varphi d \chi \quad \text { for } y \in \bar{W} .
$$


Next, consider the map

$$
d: N \rightarrow R \times W, \quad(x, a) \mapsto(\langle x, x\rangle, a) .
$$

By [P-S] (cf. Theorem 2.4) the function $d_{*} \pi^{*} \varphi$ is algebraic. Therefore, we are reduced to the situation that $\varphi$ lives on $R \times W$, and it remains to show that $r_{*} \varphi$ is generically algebraic on $\bar{W}$.

4. As before, we also consider $\bar{R} \times \bar{W}$. We may assume that $\varphi=\operatorname{sign}(f)$ with $f \in R[W][X]$. Since we need only show that $r_{*} \varphi$ is generically algebraic on $\bar{W}$, instead of looking at real points on $\bar{W}$ we look at orderings $\bar{\alpha} \in$ $\operatorname{Spec}_{\mathrm{r}}(\bar{R}(\bar{W}))$, that is, we will show that $r_{*}^{\sim} \varphi \mid \operatorname{Spec}(\bar{R}(\bar{W}))$ is algebraic. As in step 1 we take $f$ to be defined over $B$ but with coefficients not all in $m$, and consider $\bar{f} \in \bar{R}[\bar{W}][X]$. We write $\bar{f}=X^{k} \bar{g}$ such that $\bar{g}$ does not vanish identically on $\bar{W}$. If $\bar{f}=\bar{g}$, then $r_{*} \varphi=\operatorname{sign}(\bar{g})$ on $\bar{W}$, up to a set of smaller dimension. So, in this case, we are done. Now, if $k>0$, consider an ordering $\bar{\alpha} \in \operatorname{Spec}_{\mathrm{r}}(\bar{R}(\bar{W}))$. Recall the situation of Remark 4.5. There is a unique ordering $\alpha \in \operatorname{Spec}_{\mathrm{r}}(R(W))$ such that $\widetilde{\varrho}(\alpha)=\bar{\alpha}$. Consider the field $R_{\alpha} \supset R$ and its valuation ring

$$
B_{\alpha}:=\left\{x \in R_{\alpha} \mid \exists b \in B: x \leq b\right\} .
$$

Let $m_{\alpha}$ be the corresponding maximal ideal. Let $f=f_{1} f_{2}^{2}$, where $f_{1}, f_{2}$ are mutually prime and square free (as elements of $R(W)[X]$ ). With these notations we have

$$
(-1)^{\operatorname{dim}(\bar{W})} \varrho_{*}^{\sim} \varphi(\alpha)=\int_{m_{\alpha}} \operatorname{sign}\left(f_{1}\right) d \chi-\#\left\{x \in m_{\alpha} \mid f_{2}(x)=0\right\} .
$$

By Proposition 4.3 and Remark 4.5 the second summand is algebraic on $\operatorname{Spec}_{\mathrm{r}}(\bar{R}(\bar{W}))$. For the first summand, we assume that $f=f_{1}$ and write $\bar{f}=X^{k} \bar{g}$ as above. Then, by Lemma 5.2 ,

$$
\int_{m_{\alpha}} \operatorname{sign}(f) d \chi=-\bar{g}(\alpha)-\sum_{2 \leq 2 k \leq \operatorname{deg}(f)} Z_{2 k, \alpha}
$$

where

$$
\begin{aligned}
Z_{i, \alpha} & :=\sum_{x \in m_{\alpha} \cap V_{k, \alpha}} \operatorname{sign}\left(f^{(i)}(x)\right), \\
V_{k, \alpha} & :=\left\{x \in R_{\alpha} \mid f(x)=\cdots=f^{(k-1)}(x)=0\right\} .
\end{aligned}
$$

Now clearly $\bar{g}(\alpha)$ is algebraic on $\operatorname{Spec}_{\mathrm{r}}(\bar{R}(\bar{W}))$ and by Proposition 4.4 and Remark 4.5 this also holds for the functions $\alpha \mapsto Z_{2 k, \alpha}$.

6. Limits. For $x \in \mathbb{R}^{n}$ and $r \in \mathbb{R}, r>0$, let $B^{n}(x, r):=\left\{y \in \mathbb{R}^{n} \mid\right.$ $\|y-x\|<r\}$, where \|\| denotes the euclidean norm.

Notation 6.1. For a constructible function $\varphi: \mathbb{R}^{n} \rightarrow \mathbb{Z}$ we denote by $\widehat{\varphi}: \mathbb{R}^{n} \rightarrow \mathbb{Z}$ the function 


$$
x \mapsto \lim _{r \rightarrow 0} \int_{B^{n}(x, r)} \varphi d \chi .
$$

Clearly, $\widehat{\varphi}$ is again constructible. Moreover, it is well known that $\widehat{\widehat{\varphi}}=\varphi$ (see [Br3]) and if $\varphi$ is algebraic, then so is $\widehat{\varphi}$. The latter follows, for instance, from $[\mathrm{P}-\mathrm{S}]$ and Hardt's Theorem $[\mathrm{Ha}]$ (see also [MC-P]). Now let $\left(\varphi_{m}\right)_{m \in \mathbb{N}}$ be a sequence of constructible functions $\varphi_{m}: \mathbb{R}^{n} \rightarrow \mathbb{Z}$.

Definition 6.2. We say that $\left(\varphi_{m}\right)$ tends to $\psi: \mathbb{R}^{n} \rightarrow \mathbb{Z}$ and write $\left(\varphi_{m}\right) \rightarrow \psi$ if for all $x \in \mathbb{R}^{n}$ one has

$$
\widehat{\psi}(x)=\lim _{r \rightarrow 0} \lim _{m \rightarrow \infty} \int_{B^{n}(x, p)} \varphi_{m} d \chi .
$$

For characteristic functions of closed convex sets this is the same as Hausdorff convergence, but in general, this is not true.

EXAMPlE 6.3. Consider the sequence of ellipses

$$
E_{m}=\left\{x \in \mathbb{R}^{2} \mid x_{1}^{2}+m^{2} x_{2}^{2}=1\right\}
$$

which get more and more flat for increasing $m$. The Hausdorff limit of $\left(E_{m}\right)$ is the interval $I=\left\{x \in \mathbb{R}^{2} \mid x_{1}^{2} \leq 1, x_{2}=0\right\}$. Now let $\varphi_{m}=1_{E_{m}}$. Then $\left(\varphi_{m}\right) \rightarrow \psi$ where

$$
\psi(x)= \begin{cases}2 & \text { for } x_{1}^{2}<1, x_{2}=0 \\ 1 & \text { for } x_{1}^{2}=1, x_{2}=0 \\ 0 & \text { else }\end{cases}
$$

Note that the $\varphi_{m}$ are algebraic and so is $\psi$, but $1_{I}$ is not algebraic. This is a special case of a general result.

Consider $\mathbb{R}^{n+k}=\mathbb{R}^{n} \times \mathbb{R}^{k}$ as a family of spaces $\mathbb{R}^{n}$ with parameters in $\mathbb{R}^{k}$. We denote the variables in $\mathbb{R}^{n}$ by $X=\left(X_{1}, \ldots, X_{n}\right)$ and those in $\mathbb{R}^{k}$ by $T=\left(T_{1}, \ldots, T_{k}\right)$. For $t \in \mathbb{R}^{k}$ let $\mathbb{R}_{t}^{n}:=\mathbb{R}^{n} \times\{t\} \subset \mathbb{R}^{n+k}$. Now let $\varphi: \mathbb{R}^{n} \times \mathbb{R}^{k} \rightarrow \mathbb{Z}$ be constructible. Again we consider $\varphi$ as a family $\left\{\varphi_{t} \mid t \in \mathbb{R}^{k}\right\}$ of constructible functions $\mathbb{R}^{n} \rightarrow \mathbb{Z}$ where $\varphi_{t}(x):=\varphi(x, t)$.

More generally, let $\alpha \in \operatorname{Spec}_{\mathrm{r}}(\mathbb{R}[T])$. Recall that we may represent $\alpha$ by a homomorphism $\alpha: \mathbb{R}[T] \rightarrow R_{\alpha}$ where $\mathbb{R} \subset R_{\alpha}$ is a real closed field. By model completeness of the theory of real closed fields, $\varphi$ induces a constructible function $\varphi_{\alpha}: R_{\alpha}^{n} \rightarrow \mathbb{Z}$.

It may be helpful to "visualize" this. It is well known and used before that to $\alpha$ there corresponds an ultrafilter $A$ of semialgebraic sets $S \subset \mathbb{R}^{n}$. Then $R_{\alpha}$ is represented by semialgebraic functions $\widetilde{f}: S \rightarrow \mathbb{R}$ for $S \in A$ where two such functions $\widetilde{f}$ and $\widetilde{g}$ are identified if they coincide on some $T \in A$. Now, 
given $\varphi: \mathbb{R}^{n} \times \mathbb{R}^{k} \rightarrow \mathbb{Z}$ it is clear that $\varphi$ assigns eventually a well defined value to an $n$-tuple $f=\left(f_{1}, \ldots, f_{n}\right) \in R_{\alpha}^{n}$.

The canonical valuation ring $B_{\alpha}$ of $R_{\alpha}$ is represented by those $\tilde{f}$ which are bounded on some $T \in A$, and the maximal ideal $m_{\alpha}$ by those $\widetilde{f}$ which are arbitrarily small on some $T \in A$. We may also write

$$
\begin{aligned}
B_{\alpha} & =\left\{f \in R_{\alpha}|\exists r \in \mathbb{R}:| f \mid \leq r\right\}, \\
m_{\alpha} & =\left\{f \in R_{\alpha}|\forall r \in \mathbb{R}, r>0:| f \mid \leq r\right\} .
\end{aligned}
$$

Note that the residue field is isomorphic to $\mathbb{R}$.

Now let $\left(t_{m}\right)$ be a sequence in $\mathbb{R}^{k}$. Then we get a sequence $\left(\varphi_{m}\right):=\left(\varphi_{t_{m}}\right)$ of constructible functions $\varphi_{m}: \mathbb{R}^{n} \rightarrow \mathbb{Z}$. So this is a sequence of constructible functions in the family $\mathbb{R}^{n} \times \mathbb{R}^{k}$. In this situation we have

Theorem 6.4.

(a) There is an element $\alpha \in \operatorname{Spec}_{\mathrm{r}}(\mathbb{R}[T])$ with corresponding ultrafilter $A$ such that for all semialgebraic sets $S \in A$ one has $t_{m} \in S$ for infinitely many $m$.

(b) Assume that $\left(\varphi_{m}\right) \rightarrow \psi$ and let $\alpha$ be as in (a). Then $\widehat{\psi}=\varrho_{*} \varphi_{\alpha}$, where $\varrho$ is the reduction map according to the real closed field $R_{\alpha}$ with the valuation ring $B_{\alpha}$. In particular, $\psi$ is constructible.

(c) If $\varphi$ is algebraic, then so is $\psi$.

Proof. (a) The collection of all semialgebraic sets $S \subset \mathbb{R}^{k}$ for which $t_{m} \in S$ for almost all $m \in \mathbb{Z}$ forms a filter $F$. Let $A \supset F$ be any ultrafilter of semialgebraic sets in $\mathbb{R}^{k}$ and $\alpha$ the corresponding element in $\operatorname{Spec}_{\mathrm{r}}(\mathbb{R}[T])$. Then clearly $\alpha$ has the required property.

(b) Let $x \in \mathbb{R}^{n}$ and $f \in R_{\alpha}$. Then $f-x \in m_{\alpha}$ if and only if for all $r>0$, all $S \in A$ and all representatives $\widetilde{f}_{i}: S \rightarrow \mathbb{R}$ for $f_{i}$ there exists $T \subset S$ with $T \in A$ such that $\widetilde{f}(t) \in B(x, r)$ for all $t \in T$. In particular, there are infinitely many $t_{m}$ such that $\widetilde{f}\left(t_{m}\right) \in B(x, r)$. We have

$$
\varrho_{*} \varphi(x)=\int_{f-x \in m_{\alpha}} \varphi_{\alpha}(f) d \chi .
$$

We may compute the right hand side by a finite procedure, say, a cell decomposition which still works on representatives defined over some $T \in A$. If we specialize the computation at some $t \in T$ we get the same value $\varrho_{*} \varphi(x)$ for the Euler integral on the one hand, but on the other hand, since there are infinitely many $t_{m} \in T$ for all $T \in A$ we get the value

Thus $\varrho_{*} \varphi(x)=\widehat{\psi}(x)$.

$$
\lim _{r \rightarrow 0} \lim _{m \rightarrow \infty} \int_{B^{n}(x, r)} \varphi_{m} d \chi .
$$

(c) This follows from (b) and Theorem 5.1. 
REMARK 6.5. In the preceding theorem, the statement that the limit $\psi$ of the sequence $\varphi_{n}$ is again constructible should also be true in the o-minimal context.

\section{References}

[A-B-R] C. Andradas, L. Bröcker and J. M. Ruiz, Constructible Sets in Real Geometry, Ergeb. Math. Grenzeb. 33, Springer, 1996.

[Be-Br] E. Becker and L. Bröcker, On the description of the reduced Witt ring, J. Algebra 52 (1978), 328-346.

[B-C-R] J. Bochnak, M. Coste et M. F. Roy, Géométrie algébrique réelle, Ergeb. Math. Grenzgeb. 12, Springer, 1987.

[Br1] L. Bröcker, On the reduction of semialgebraic sets by real valuations, in: Contemp. Math. 155, Amer. Math. Soc., Providence, RI, 1994, 75-95.

[Br2] - Families of semialgebraic sets and limits, in: Real Algebraic Geometry (Rennes, 1991), Lecture Notes in Math. 1524, Springer, 1992, 145-162.

[Br3] -, Euler integration and Euler multiplication, Adv. Geom. 5 (2005), 145-169.

[Ha] R. Hardt, Semi-algebraic local-triviality in semi-algebraic mappings, Amer. J. Math. 102 (1980), 291-302.

[L-S] J.-M. Lion and P. Speissegger, A geometric proof of the definability of Hausdorff limits, preprint, 2003.

[MC-P] C. McCrory and A. Parusiński, Algebraically constructible functions, Ann. Sci. École Norm. Sup. 30 (1997), 527-552.

[P-S] A. Parusiński and Z. Szafraniec, On the Euler characteristic of fibres of real polynomial maps, in: Singularities Symposium-Łojasiewicz 70, Banach Center Publ. 44, Inst. Math., Polish Acad. Sci., Warszawa, 1998, 175-182.

[vdD1] L. van den Dries, Tame Topology and o-Minimal Structures, Cambridge Univ. Press., 1998.

[vdD2] - Hausdorff limits of definable spaces, preprint, 2003.

[V] O. Y. Viro, Some integral calculus based on Euler characteristic, in: Topology and Geometry-Rohlin Seminar, Lecture Notes in Math. 1346, Springer, 1988, $127-138$.

Mathematisches Institut

Einsteinstraße 62

D-48149 Münster, Germany

E-mail: broe@math.uni-muenster.de

Reçu par la Rédaction le 25.11.2004

Révisé le 9.5.2005 